\title{
DESIGNING REMOTE PLACES IN THE POST-WAR AND PANDEMIC SCENARIOS. SMART SURVEYING OF THE GAHAYR CAMPUS IN MOGADISHU
}

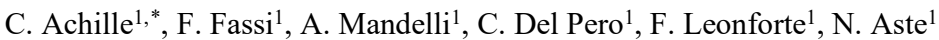 \\ ${ }^{1}$ Architecture, Built environment and Construction engineering (A.B.C.) Department, Politecnico di Milano, Milano, Italy - \\ (cristiana.achille, francesco.fassi, alessandro.mandelli, fabrizio.leonforte, claudio.delpero, niccolo.aste)@polimi.it
}

KEY WORDS: heritage documentation, remote documentation, 3D survey, photogrammetry, capacity building

\begin{abstract}
:
The study presents a part of the operational framework of the "Project for Infrastructure and Strategic Strengthening of the Somali National University", funded by the Italian Agency for Development Cooperation. The project, coordinated by Politecnico di Milano, aims to reconstruct the Gahayr campus of the Somali National University of Mogadishu, which is today almost destroyed due to the civil war. The preliminary phase for reconstruction is a detailed survey of the buildings and the area over which the Campus will be re-built. In a normal situation, the team in charge of the survey would have gone on-site in Mogadishu; nevertheless, the risky local conditions and the Covid-19 pandemic made it impossible to have foreign personnel on-site. Consequently, the choice was to train a local team remotely, giving them the theoretical and practical instruments to face a complete 3D survey of the area and the buildings. Harsh times cannot stop works and activities that usually need the presence of the survey team on the field. Careful planning of the activities, the online staff training and the continuous sharing of the information permitted to get high quality $3 \mathrm{D}$ metric results quickly and to have at disposal all dimensional and qualitative valuable information for the project, usable in real-time by the designers and architects without going directly on the site.
\end{abstract}

\section{GAHAYR CAMPUS. A BRIEF INTRODUCTION}

The construction of the Gahayr Campus, headquarters of the Somali National University, started in the early '70s with the donation by Italy of 90 hectares of land in the western area of Mogadishu, following a specific cooperation agreement between the Italian Government and the Federal Transitional Government of Somalia. The project was developed by Architects Lodovico Quaroni and Salvatore Dierna and adapted to the specific needs of the context by Architect Roberto De Fraia, sent on-site to support the Somali Construction Authority in the implementation phase. The Campus has a concentric arrangement and includes a central core for the rectorate, buildings for the departments and classrooms, services and housing for students and teachers. It was officially opened in 1985. The European Development Fund financed the construction. The structure remained in operation until the outbreak of civil war in January 1991. It was later formally reopened on 16 August 2014, after suffering heavy damage and looting.

Nowadays, the Gahayr Campus appears in a critical state; many buildings have been demolished, while the remaining ones are in an advanced state of degradation. The remaining empty spaces have been occupied by the growth of the surrounding urban fabric in the form of illegal slums (Figure 1).

In such a context, the aim of the "Project for Infrastructure and Strategic Strengthening of the Somali National University", financed by the Italian Agency for Development Cooperation (AICS, 2021) and coordinated by Politecnico di Milano, is the planning of the restoration of the remaining buildings, the design of the new rectorate and, above all, the development of a new masterplan that will guide the reconstruction and expansion of the entire premises.

The new Gahayr Campus will include the rectorate, seven faculties, residences for students and teachers, services, sports facilities, and open spaces for agricultural and zootechnical activities. According to progressive logic, its construction is planned up to host in the final configuration about 20,000 students.

The project under development is multidisciplinary and aims to provide technologically advanced and sustainable answers and solutions to the current and future needs of the National Somali University, strengthening the profound link between the Italian and Somali academic world. More in detail, among the constructions not destroyed, the seven faculty buildings of the Gahayr Campus represent the last legacy of the original project. As their fabrication had it in the previous century, their renovation has today a symbolic value besides a practical and functional one. They are the testimonies of the deep link between Italian and Somali culture and the demonstration of the cooperation over time between the two countries. For these reasons, within the Infrastructural and Strategic Strengthening of the Somali National University framework, the functional and architectural refurbishment of the faculty buildings is considered paramount.

The retrofit design concept, developed by Politecnico di Milano, aims to guarantee environmental, energy and economic efficiency, to set a benchmark for the spread of sustainable architecture in Somalia.

A comprehensive feasibility study for the rehabilitation and functional reuse was focused on building number 4 , which the Faculty of Engineering will use. Considering the strong similarities, the results can also be extended to the other buildings. First, it must be noted that preserving the original design, the new one is based on the substantial refurbishment of the initial building configuration. Therefore, the floor plan and the internal walls have been not significantly modified, except for merging some classrooms to obtain larger spaces.

Thus, a detailed, comprehensive, and precise architectural/structural survey of the building is crucial to the definition of retrofit interventions.

\footnotetext{
${ }^{*}$ Corresponding author
} 

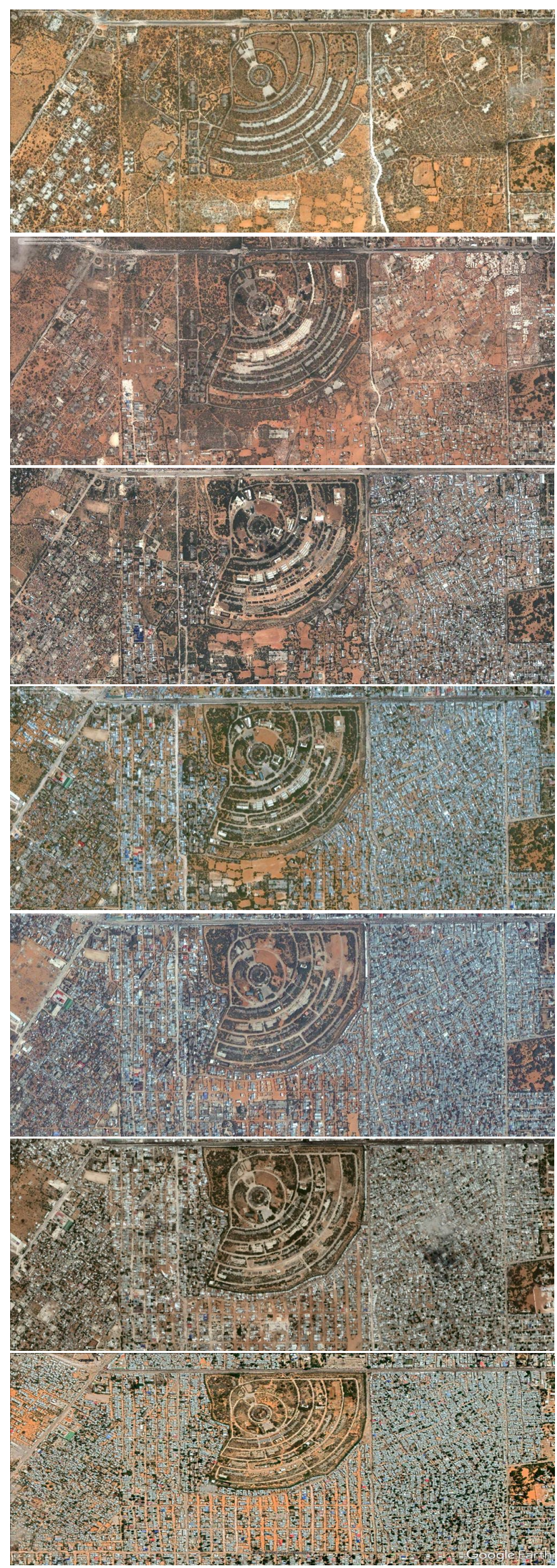

Figure 1. Google Earth images from 2006 to 2020 show the impressive development of the urban fabrics and illegal slums.
However, the critical and challenging application context does not allow foreign personnel to be sent to the site for security reasons; this strongly limits the possibility to assess the status of the constructions, with the risk of introducing erroneous evaluations.

\section{THE SURVEY OF THE GAHAYR CAMPUS}

\subsection{Preliminary observations}

The preliminary phase for the reconstruction is a detailed survey of the buildings and the area over which the new Campus will be built. The form of the Campus area is approximately a square with an edge of about 600 meters. The terrain's morphology is irregular and covered by shrub vegetation; the rectorate building is at a higher position to the area's borders; the height difference is about 20 metres. The requirements were a general map of the whole area and cross-sections of the site at 1:200 scale to support the masterplan design, and a similar approach was also studied in Sedláček et al. (2020)

For the refurbishment, detailed maps of the inner parts of the buildings at 1:100 scale were produced. The interior survey was also intended to show the condition of the structures and facilitate the structural interpretation.

The ideal solution to guarantee the requests was a complete $3 \mathrm{D}$ survey of the whole area integrating different instruments to represent both external and internal parts from a geometric dimensional and chromatic point of view.

As discussed in Gulec Korumaz et al. (2014) and Caprioli et al. (2015), photogrammetry with UAV and Laser Scanner would have been the classical survey solution adopted in a normal situation after a preventive mission to evaluate the state of conservation of the place and plan the necessary survey actions. Nevertheless, the Covid-19 pandemic makes it impossible to travel abroad. Moreover, working in Somalia requires caution for foreign people, both for health and security reasons.

The problem to be solved is how to entrust a complete survey at medium-large scales of representation that requires expensive instrumentation, dedicated training and experience in data processing. The choice pursued was to develop a survey pipeline that would use easy and fully automatic tools for both the acquisition and processing of raw data, but that would guarantee completeness, reliability, low cost and the possibility of cloudinterfacing data in input and output. Automatic data processing, simple instructions, data sharing and dedicated remote training were the keys to the project's success.

The idea was to use a drone with autonomous capabilities to survey the environment of the whole area to extract the DTM (Digital Terrain Model) with $2 \mathrm{~cm}$ resolution and extract a detailed orthophoto of the site.

The Matterport Pro 2 3D camera was selected to survey the interiors of the buildings with triple goals i) to extract semiautomatically the plans, ii) to have at disposal a raw point cloud model to extract additional measurements and iii) to create a complete virtual tour of the site automatically at disposal for the stakeholder to perform the diagnostic survey.

A local company with previous experience in classical topographic surveys was selected and trained remotely, giving them the theoretical and practical instruments to face the complete 3D survey of the terrain and the buildings following the designed pipeline.

The local company performed additional measurements: GNSS antenna measurement to georeference and scale the photogrammetric data and total station survey to complete the building survey. 


\subsection{UAV and GNNS survey}

For the general survey of an extensive area in a relatively short time and in an automatic way, the use of a UAV (Unmanned Aerial Vehicle) is nowadays a natural choice. Thanks to performing camera sensors of actual commercial drones, it is possible to get high resolution and high-quality results using lowcost devices. The possibilities to use a UAV able of autonomous flight allow designing the ideal flight and planning the position of few markers to guarantee a reliable photogrammetric result, as demonstrated in De Reu et al. (2016), Federman et al. (2017), Adami et al. (2019) and Jiang et al. (2020).

Analysing commercial drones' market and evaluating the camera sensors and the flight performances, the DJI Mavic 2 PRO was chosen. In the same way, some navigation software was investigated considering the drone manufacturers supported, the system's reliability, compatibility with different platforms, and price. This search led to the choice of FlyLitchi. It integrates an online mission hub platform that lets the operator create virtual flight missions using a background satellite layer from Google Maps. The mission is designed by placing up to 99 waypoints on the map; for each waypoint, the user defines: i) the altitude, ii) the drone's speed, iii) the action performed by the drone, iv) the angle between the camera and the terrain. Once the mission is created and saved, the same mission can be shared on the cloud and used with a mobile device, both Android or iOS and then uploaded to the drone. The solution satisfies the requirements: the ease of use of the system, a brief but exhaustive remote training of the local company, the possibility to plan the flight remotely and, generally, reset the chance of error in execution. The missions were designed in Milan at Politecnico, the dimensions of the area to be covered with the photogrammetric acquisition and the level of detail required by the designers of the Campus lead to plan four different flights. The design of the missions considered the following parameters: i) 1:200 drawing scale, both for the orthophoto and the cross-sections, ii) the resolution of the camera, iii) the focal length, iv) the maximum altitude reachable by law, v) the time of the mission, vi) the longitudinal overlap equal to $80 \%$, and vii) the transversal overlap equal to $60 \%$. The DJI Mavic 2 PRO is equipped with a CMOS 1" sensor with a resolution of $5.472 \times 3.648$ pixels, the pixel size is $2,4 \mu \mathrm{m}$, and the focal length is $10 \mathrm{~mm}$. The maximum flight autonomy declared by the manufacturer is 31 minutes. The Ground Sample Distance (GSD) calculated for the 1:200 drawing scale is $2 \mathrm{~cm}$. The design of the flights starts from the proportion:

$$
\begin{gathered}
c: H=p x: G S D \\
H=\sim 80 \mathrm{~m}
\end{gathered}
$$

where: $\quad c=$ principal distance

$$
\begin{aligned}
& c=\text { principal distance } \\
& H=\text { height of the flight } \\
& p x=\text { pixel size } \\
& G S D=\text { Ground Sample Distance }
\end{aligned}
$$

It was decided to perform flights at 70 meters from the ground except for a diagonal strip that connects the last waypoint with the starting point at the height of $80 \mathrm{~m}$. At this height, the theoretical value of the GSD is equal to $1,68 \mathrm{~cm}$. The role of the diagonal stripe is to strengthen the aerial photogrammetric block. The second step was to calculate the footprint on the terrain of a single frame acquired from $70 \mathrm{~m}$; the result is a rectangle of 91 $\mathrm{m}$ by $61 \mathrm{~m}$. The longitudinal overlap depends on the interval between each shot and the cruising speed of the drone. The $80 \%$ overlap is equal to $48,8 \mathrm{~m}$; this means having a capture-base of circa $12 \mathrm{~m}$. It is possible to calculate the necessary cruising speed assuming 2 seconds between the first and the following.

$$
\begin{gathered}
B=v * \Delta t \quad B=\frac{H * l *(1-\mu)}{c} \\
v * \Delta t=\frac{H * l *(1-\mu)}{c} \\
v=6 \mathrm{~m} / \mathrm{s}
\end{gathered}
$$

where: $\quad B=$ base distance

$v=$ cruising speed

$\Delta t=$ shooting interval

$H=$ height of the flight

$l=$ sensor length

$\mu=$ longitudinal overlap

$c=$ principal distance

The transversal overlap depends on the distance between the strips. To assure an overlap of $60 \%$, this distance was imposed equal to $25 \mathrm{~m}$. The last parameter to be considered is the dragging effect on the frames due to the drone's motion during the acquisition phase. This effect depends on the focal length, the cruising speed, the height of the flight, and the shutter speed that was equal to $1 / 200 \mathrm{~s}$ for each image. The formula to calculate the dragging effect is the following:

$$
\begin{gathered}
D r=\frac{c * v * t}{H} \\
D r=0,0031 \mathrm{~mm}(\text { on the sensor) } \\
D r=2,5 \mathrm{~cm} \text { (on the ground) }
\end{gathered}
$$

where: $\quad D r=$ dragging effect

$c=$ principal distance

$v=$ cruising speed

$t=$ shutter speed

$H=$ height of the flight

The result of the dragging effect was considered acceptable because it is lower than the GSD designed at the beginning of the survey, evaluating the 1:200 drawing scale. Once all the parameters were defined, it was possible to draw the mission plan map (Figure 2). The time of the mission is automatically updated each time a waypoint is placed on the map. The area of the Campus was subdivided into four regions according to the time needed to survey them. Each mission lasted about 22 minutes. This value is compatible with the maximum autonomy of the DJI Mavic Pro 2 that is around 31 minutes in average weather conditions. The flights were performed on bright days, around midday, to prevent long shadows and in the absence of strong wind.

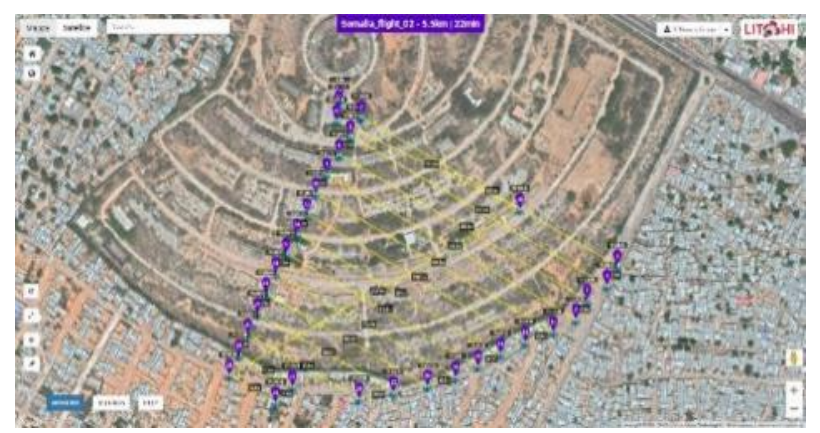

Figure 2. Flight plan n. 2. 
Lastly, even the terrain's morphology is mainly planar, a last check on the altitude was done using Virtual Litchi Mission (Figure 3). This software automatically connects Litchi Mission Hub and Google Earth. The software plots a graph with the height of the flight with respect to the ground and displays the drone's trajectory on the 3D map (Figure 4).

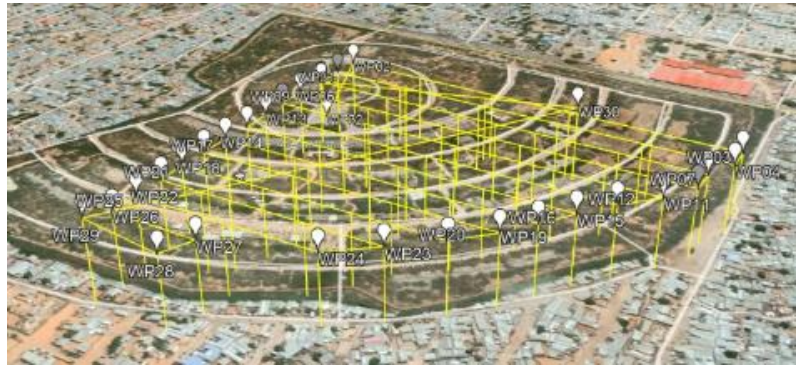

Figure 3. 3D plot on Google Earth of flight n. 2.

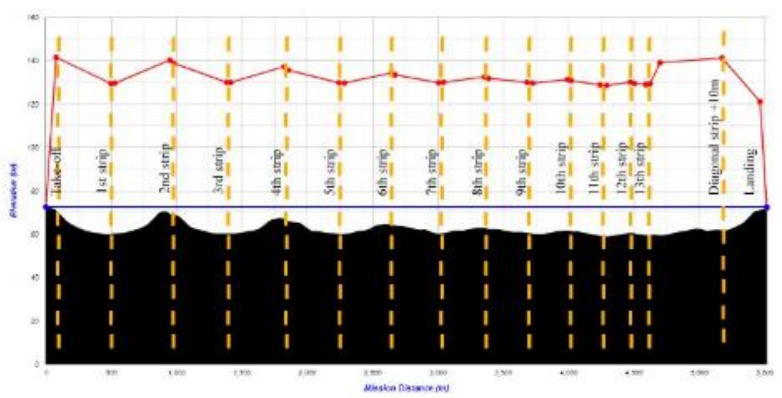

Figure 4. Path of flight n. 2, checking the altitude.
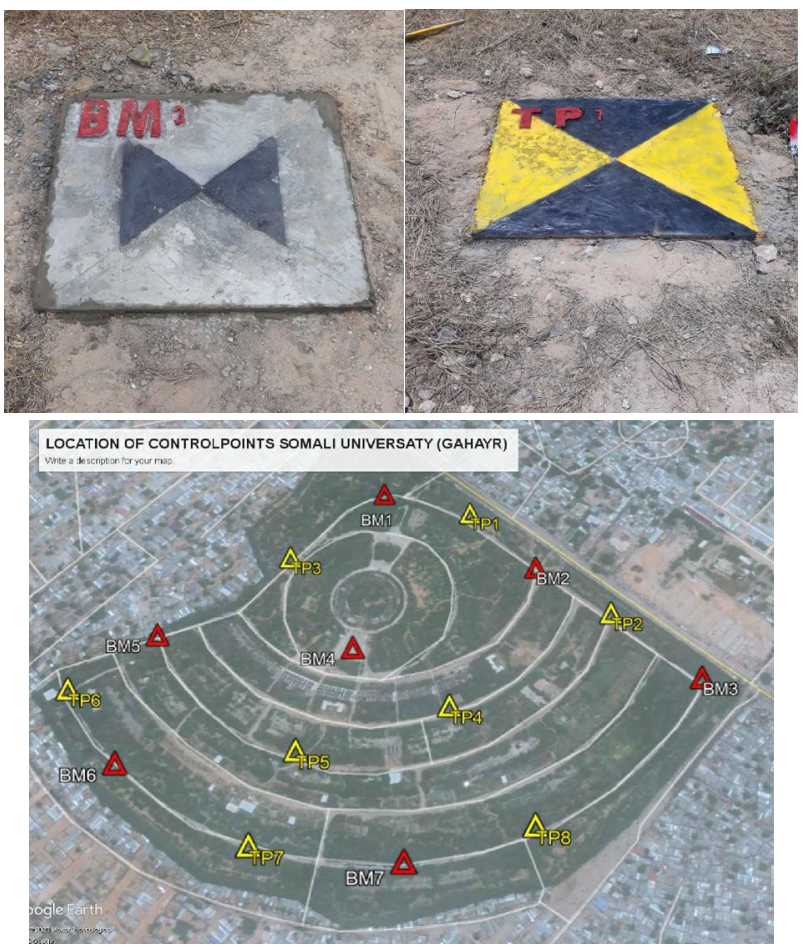

Figure 5. Location of the markers. Upper left concrete GCP, right temporary GCP.

For the scaling of the photogrammetric model and georeference in the same system for all the UAV-flights that will occur over time in the area, a series of Ground Control Points (GCPs) were measured. The coordinates of the points were determined using the GNSS survey in RTK mode, which means using a dual antenna GNSS system (reference + rover). The reference station has been positioned in the most elevated central point of the area and remained on for the survey's duration. At the same time, the other GCPs were measured with the rover station with a minimum stay on the point of 1 minute to guarantee a precision of $1 \mathrm{~cm}$ or less.

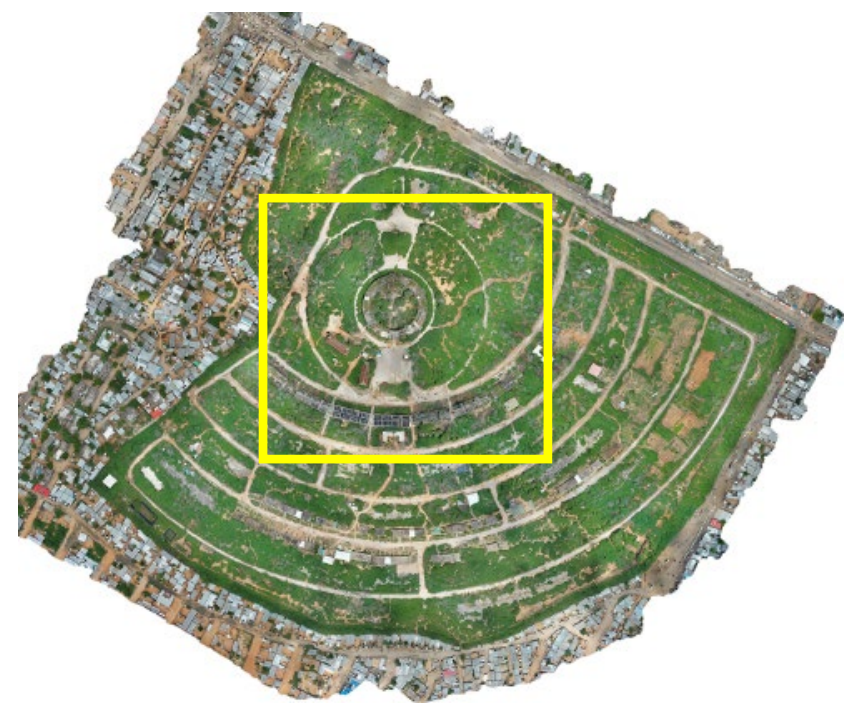

Figure 6. Orthophoto, pixel size $2 \mathrm{~cm}$.

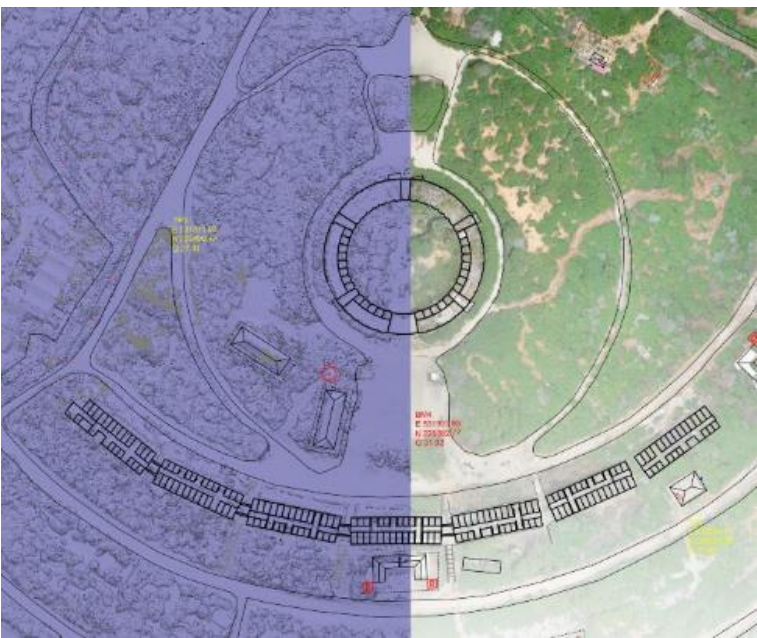

Figure 7. On the left, the high-resolution 3D model, on the right, a detail of the orthophoto, superimposed the CAD drawing digitized from the dense point cloud.

It was essential to check the satellite coverage and the relative measurement accuracy with the reference station, around or less than one centimetre of accuracy. The GCPs were placed over the area following a logical uniform distribution, as studied in Villanueva and Blanco (2019). Some markers were materialized permanently on the terrain using concrete plates directly, allowing the possibility to repeat the flight in the future to monitor the restoration and fabrications activities. They have $1 \mathrm{~m}$ $\mathrm{x} 1 \mathrm{~m}$ dimensions, and the classic black and white cross targets were painted on the concrete plate (Figure 5). At the centre of the targets, a topographic nail was positioned and surveyed topographically. Oher markers, temporary ones, were build on wood and placed in the area to constrain the photogrammetric block. 


\subsection{Gahayr orthophoto and terrain profiles}

As soon as each flight was completed, the images and GNSS coordinates were uploaded into the cloud. The elaboration was performed in Italy. A first check to verify the visibility of the markers and the quality of the photos was performed (Villanueva and Blanco, 2019). In the same way, the coordinates of the points measured with a GPS antenna were transmitted to the Politecnico di Milano, which processed all the data. The data were elaborated with Agisoft Metashape (Version 1.7 Build 12070). The project was composed of 838 images as a result of the sum of the four flights. The workflow considered the fundamental steps of the photogrammetric elaboration: i) alignment of the images, ii) manual detection of the markers, iii) optimization of the camera parameters, iv) georeferencing of the 3D data, v) computation of the dense cloud, vi) generation of the orthophoto (Figure 6 and 7). During the elaboration phases, the error on the check points was checked, which is equal to $2 \mathrm{~cm}$. The exact value was imposed as GSD for the orthophoto generation. These values are consistent with the 1:200 drawing scale designed at the beginning of the work. The project lets deliver a high-resolution orthophoto in which GSD is 2 centimetres, a dense point cloud with an accuracy of 2 centimetres and some longitudinal and transversal crossing sections. The drawings were realised using the $3 \mathrm{D}$ point cloud in AutoCAD environment and slicing it where the designers needed information to layout the new Campus's masterplan.

\subsection{Architectonical remote survey}

A different approach was used to document the buildings that are in a state of disrepair. The goal was to illustrate the conservation of buildings, especially the internal parts (structural and nonstructural parts), in a capillary and accurate manner using a lowcost device for the impossibility to ship abroad expensive and challenging to use instrumentation (such as the laser scanners). The second goal, as mentioned before, was to highlight the actual state of conservation, making available to the designers not only dimensional graphs but also a catalogue of referenced images that show the state of conservation of the structures, materials and other information that can help in the design phase.

Some evaluations were made about the equipment that could be used. Two criteria influenced the choice: the first linked to the cost of the instrumentation. The second criterion concerned the adherence of the survey data returned with the actual situations. Some buildings are in poor condition (Figure 8). The technical drawing of plans, sections and facades must highlight the actual state of conservation of the structures.

Not being able to proceed with an inspection and survey on the site, the Italian staff decided to choose the Matterport Pro 2 3D Camera (Matterport, 2021).

The 3D camera is designed to survey interior environments. It produces a virtual tour as a final output creating an immersive 3D virtual representation of the real world. The camera consists essentially of a panoramic rotating camera that mounts different sensors: a camera $360^{\circ}$ to capture the colour and depth data needed to create a 3D replica of a real-world, a more detailed description in (Pulcrano, et al., 2019, Shults et al., 2019).

This type of instrument allows you to simultaneously acquire the shape (structured light - infrared 3D sensor) and colour (Output Pano Pixels 134.2 MP, equirectangular) of the objects. The outcome of the Matterport survey is a virtual tour of the building on which it is possible to make measurements, extract images, insert tags. Matterport cannot be considered an accurate 3D measuring tool; it mainly spread in the real estate field, offering high-quality virtual tours with measuring capabilities to drive the potential buyer's decision-making (Sulaiman et al., 2020).
On the other side, the instrument is compact, handy and low cost; the acquisition is automatic, fast and very easy; the results are entirely automated, performed on a remote server with no human intervention and finally shared on the cloud. The field survey is managed through the Matterport app (tablet or mobile phone); automatically, the data are uploaded on the cloud when the survey is complete. The panoramic images and depth data are processed to obtain a dimensionally $3 \mathrm{D}$ digital twin of space. It is possible to download the data (images, screenshots, plan view, dense cloud and textured 3D models), navigate the tour, and share the data with other stakeholders from the account.

Using the cloud platform, the collaboration by the Italo-Somalo working group increase (multiple accounts), all participants can access data and actively collaborate in the production of the technical drawings. The platform permits sharing 3D models of the buildings and using them to take measures, extract floor plans, 4K HDR photos, and extract 3D Files (*.obj, *.xyz).

Being the tool expressly indicated for the survey of interior environments and with a lot of texture, it was first necessary to evaluate its applicability to the Somali case.

Before being shipped to Somalia, the instrument was tested on similar case studies to those of Mogadishu. A series of tests were conducted with different lighting and sun conditions, various colours of the objects shot, different indoor/outdoor environments with the presence/absence of targets. The data acquired with Matterport were compared with a laser scanner survey (RTC360 Leica). The comparison confirmed that the Matteroport allow producing technical drawings at a maximum 1: 100 scale.

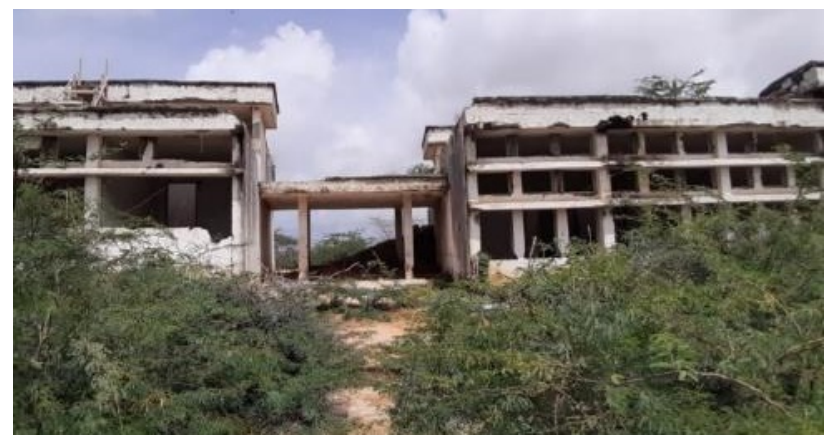

Figure 8. Two buildings of the Faculty of Engineering of the Campus.

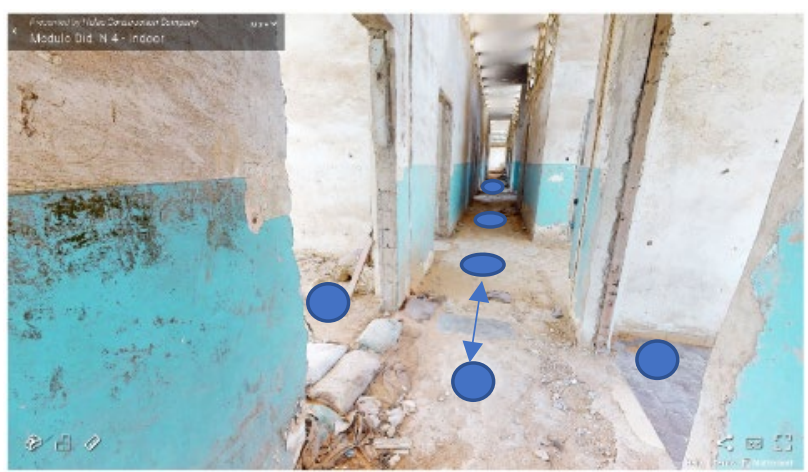

Figure 9. Different scan stations of the Matterport camera to connect the interior rooms and the corridor correctly.

Different rules have been observed to achieve a complete, correct reconstruction respecting the accuracy of the planned representation scale of 1:100. The maximum distance between two consecutive "scans" must be between 1.5 to 2.4 meters (Figure 9) to maintain a clear line of sight between the scans. Almost all the markers were placed at eye level inside the 
building, respecting Matterport suggestion (Figure 10). The number of scans varies depending on the dimension of the space. As expected, outside the light conditions make the survey more complex due to the structured light infrared sensor that does not work correctly under direct light. It was necessary to perform the survey early in the morning, avoiding extreme light differences and minimizing the direct sunlight. Despite everything, outside spaces lit entirely by direct sunlight were affected by some holes in the $3 \mathrm{D}$ model.

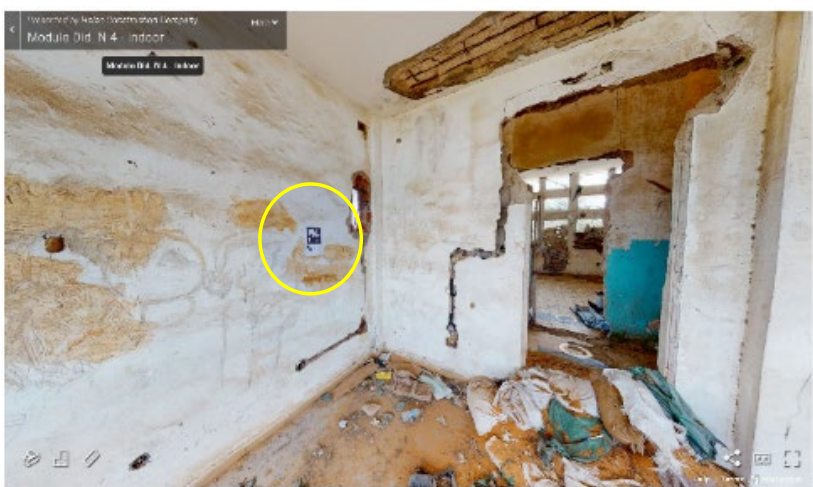

Figure 10. Some targets were placed around on the building wall to constrain the acquisition under challenging areas better.

Also, the roofs have been measured - where possible. (Figure 11). Even if the system failed to model the object in the external parts, the panoramic images could provide information about the areas and structures from a qualitative point of view, helping interpret the shapes and state of conservation of the building. Combining Matterport data and direct surveys on-site, it was possible to overcome the problem and complete the technical drawings.

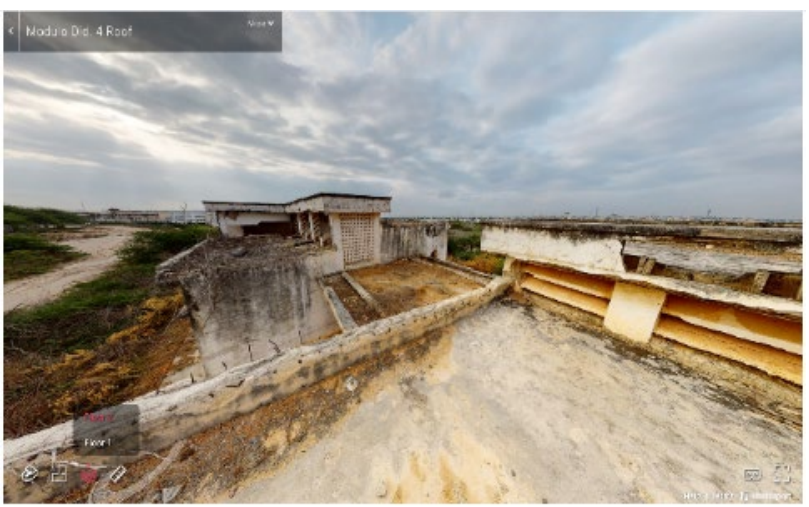

Figure 11. One image of the roof views.

In this way, even if it is not possible to take linear measurements, the high-quality panorama helps interpret the shapes and state of conservation of the building. Combining Matterport data and direct survey on-site, it was possible to fill technical sheets and draw plans and sections.

\subsection{Virtual tours and technical drawings}

Five buildings have been surveyed and are automatically visible inside the Matterport account (Figure 12). Each Campus buildings have its own dedicated Matterport space. For each space, it is possible to navigate inside the virtual tour of the building, seeing in detail what its state is and taking linear measurements, so performing a virtual direct manual measuring on the building. The available information measuring inside the virtual tour combined with the direct survey on the field made it possible to return the expected $2 \mathrm{D}$ drawings, indispensable for the subsequent design phase. Moreover, starting from the $3 \mathrm{D}$ model, a schematic floor plan can quickly and easily generate automatically by Matterport and exported in PNG and PDF files and used as an initial sketch of the final plans (Figure 13). It is necessary to underline that only parts with a geometrical $3 \mathrm{D}$ survey are included in the drawings.

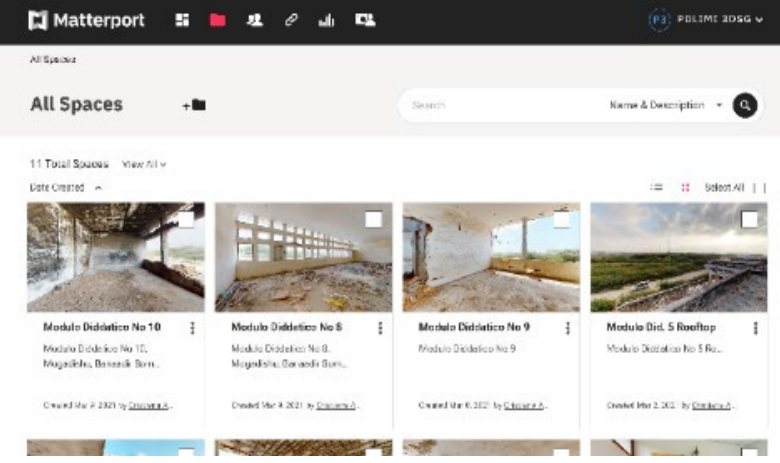

Figure 12. Online Gahayr buildings virtual tours, Matterport spaces.

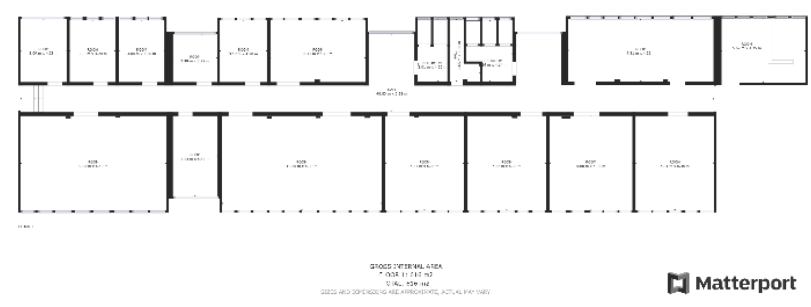

Figure 13. The Matterport schematic floor. The ground floor automatic extracted from 3D data.

The sketch returns the building shape, but unfortunately, most outside part is represented only by panorama images in the Somali case. The perimeter walls don't describe the correct position of pillars. Inside-outside many sections are broken, and the automatic plan extraction does not consider this event.

To complete sketches and drawings, it was necessary to perform a virtual manual survey directly on the $3 \mathrm{D}$ model inside the Matterport space and acquire additional data on the field with a total-station and classical accurate direct manual measurements. The final technical drawings balance measures taken on-site by local staff and measures taken via the web on the virtual tour (Figure 14). Total station and manual measurement also have the role of final check of the drawings.

The final results of elaboration are technical drawings in *.dwg format. One horizontal plan, one reverse beams plan, three vertical sections, one longitudinal and two transversal sections, four elevations, were produced (Figure 15 and 16). Each file is organized into decided/agree layers. Staff can be added layers and symbols if necessary for the accurate description of the building. The shape of the Campus buildings is not particularly complex; in normal working conditions, the drawing of plans and sections would be easy. In this case, the complexity linked to the natural working conditions and conservation of the structures, the presence of masses of debris, the dangerousness of part of the structures makes the local team's work quite challenging, in addition to the limitations mentioned above for security issues linked to the country's political instability. The tool has proved helpful for documenting the buildings briefly, allowing repetition of the survey in the time, to monitor the progress of the work remotely. This hybrid way of work (remote and on-site) has proved effective. 


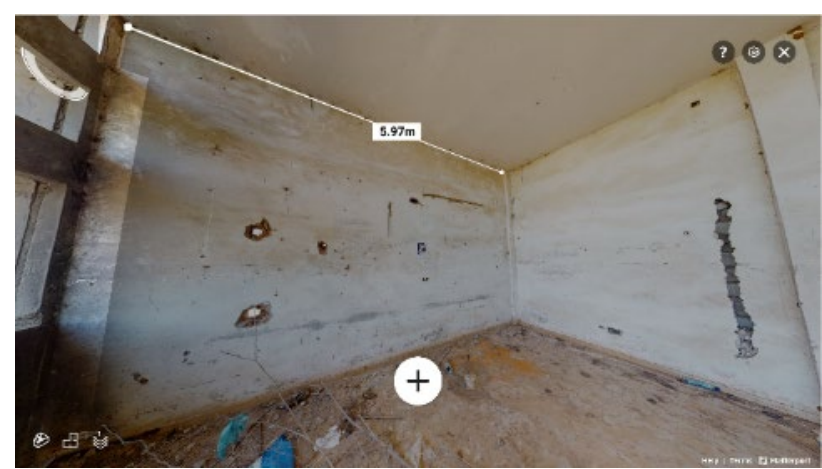

Figure 14. The measurement tool advantageous to find the dimensions of objects and rooms.

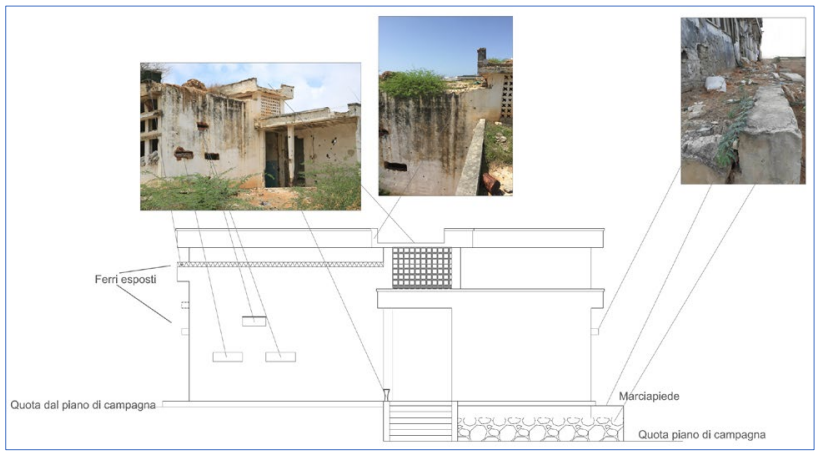

Figure 15. An example of facade drawing by Somali technical staff. Virtual tours, videos and images help the sketch phase and avoid going back on site if some information is missing.
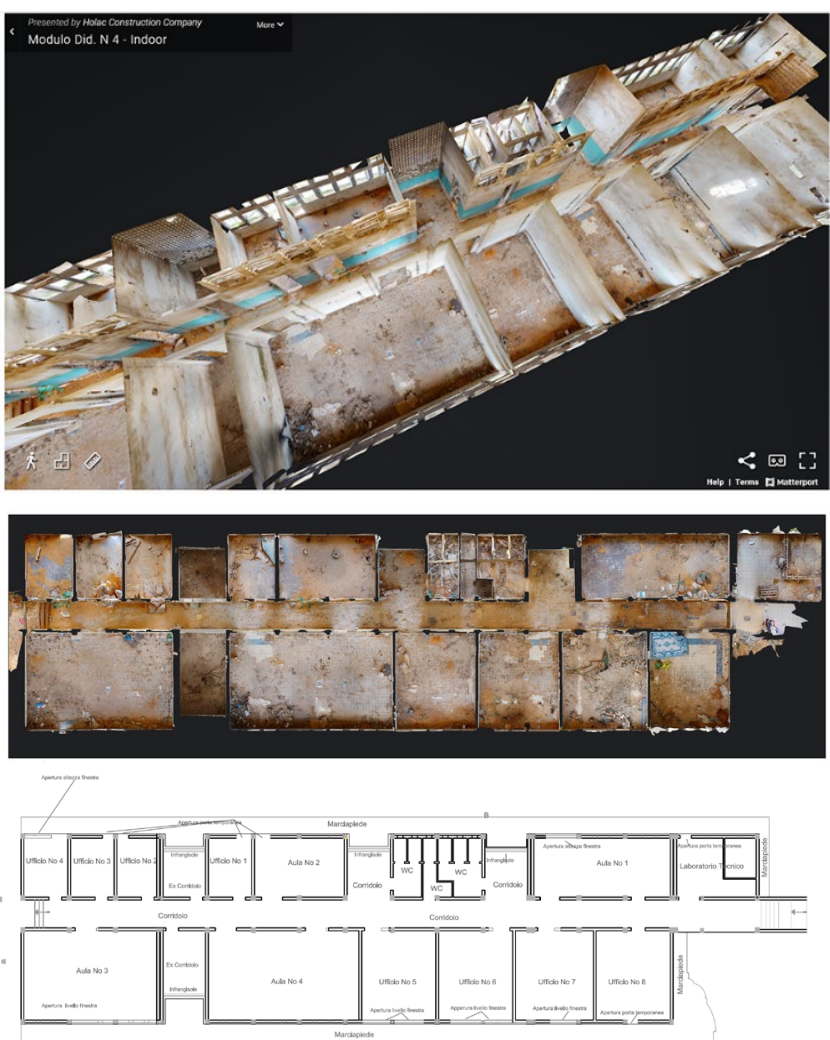

Figure 16. Matterport dollhouse and floor plan view of Building four. The simultaneous use of different 2D and 3D views combined with direct survey permits redraws the plan shape. The plan is the first technical result, and the other 2D representations arise from it.
The tool has proved helpful for documenting the buildings without interpretation. The repetition of the survey, in the same way, will allow in charge staff to monitor the progress of the work remotely. This hybrid way of work (remote and on-site) has proved effective.

\section{CONCLUSIONS}

This project stage is the complete survey of eight buildings inside the university Campus to get the comprehensive geometry knowledge of the area and allow a remote investigation of their state of conservation and a visual structural survey.

The produced information (orthophoto, 3D models and virtual tours) allows shared remote navigation of the environments and enables all project teams to simultaneously virtual visit the area and cooperate in analysing the environment, annotating, sharing comments and recommendations, and ides with all the operators. The Somali delicate political situation and worldwide healthcare situation did not allow regular activity and standard procedures (survey, design, construction, ...), with the risk of seriously compromising the entire project's success.

Only digital cooperation between countries and people was possible/fundamental to reach the scope even in these difficult circumstances.

New surveys and digital technologies always more reasonable, and the increasing possibility of universal, secure, inclusive, and affordable access to the Internet has made it possible to complete this work.

The effective success of international projects also depends on high-quality planning and targeted capacity-building activities. Careful planning of the activities joined with the online staff training and the continuous sharing of the information permitted to get high-quality $2 \mathrm{D}$ and $3 \mathrm{D}$ metric results. The project demonstrates the need for digital capacity building as an absolute prerequisite for achieving real and sustained progress.

Culture and education are essential factors for achieving stability after conflicts and disasters. Working closely with local civil society is indispensable and allows realistically evaluating the duration and feasibility of projects (Rufian 2020, UNESCO, 2014). From this point of view, the constant exchange between Italian and Somali teams permits each other to understand problems better and find share solutions.

The Project for Infrastructure and Strategic Strengthening of the Somali National University can be described as an excellent example of digital cooperation. We hope it can be a significant milestone in achieving better digital cooperation globally.

\section{ACKNOWLEDGEMENTS}

The work presented is part of the Project Infrastructural and strategic strengthening of the Somali National University Reconstruction of the Gahayr Campus in Mogadishu, funded by AICS - Italian Agency for Development Cooperation and coordinated by Politecnico di Milano (Prof. Niccolò Aste, Department of Architecture, Built Environment and Construction Engineering).

The authors thank the Somali local technical team for the effective collaboration.

\section{REFERENCES}

AICS, Agenzia Italiana per la Cooperazione allo Sviluppo, 2021, June 2021, https://www.aics.gov.it/wpcontent/uploads/2021/02/PoliMI-UNS-Booklet.pdf

Adami, A., Fregonese, L., Gallo, M., Helder, J., Pepe, M., Treccani, D., 2019. Ultra-light UAV systems for the metrical 
documentation of cultural heritage: applications for architecture and archaeology. Int. Arch. Photogramm. Remote Sens. Spat. Inf. Sci. XLII-2W17, 15-21. https://doi.org/10.5194/isprs-archivesXLII-2-W17-15-2019.

Caprioli, M., Mancini, F., Mazzone, F., Scarano, M., Trizzino, R., 2015. UAV Surveys for Representing and Document the Cultural Heritage, in: Le Vie Dei Mercanti - XIII Forum Internazionale Di Studi - HERITAGE and TECHNOLOGY Mind Knowledge Experience. Aversa-Capri, Italy, pp. 476-483.

De Reu, J., Trachet, J., Laloo, P., De Clercq, W., 2016. From Low-Cost UAV Survey to High Resolution Topographic Data: Developing our Understanding of a Medieval Outport of Bruges. Archaeol. Prospect. 23, 335-346. https://doi.org/10.1002/arp.1547.

Federman, A., Santana Quintero, M., Kretz, S., Gregg, J., Lengies, M., Ouimet, C., Laliberte, J., 2017. UAV photgrammetric workflows: A best practice guideline. Int. Arch. Photogramm. Remote Sens. Spat. Inf. Sci. 42, 237-244. https://doi.org/10.5194/isprs-archives-XLII-2-W5-237-2017.

Gulec Korumaz, A., Korumaz, M., Tucci, G., Bonora, V., Korumaz, A.G., Niemeier, W., Riedl, B., 2014. UAV systems for documentation of cultural heritage, in: ICONARCH IInternational Congress of Architecture-Innovative Approaches in Architecture and Planning. Konya,Turkey, pp. 419-430.

Jiang, X., Liu, J., Zhen, H., Li, L., Xu, Z., Shao, S., 2020. Application of the UAV Technology in the Topographic Map Survey, in: Lecture Notes in Electrical Engineering. Springer, pp. 1481-1487. https://doi.org/10.1007/978-981-15-32504 - 192 .

Matterport 2021: https://matterport.com/ June 2021

Pulcrano, M., Scandurra, S., Minin, G., Di Luggo, A. 2019. 3D cameras acquisitions for the documentation of Cultural Heritage, nt. Arch. Photogramm. Remote Sens. Spatial Inf. Sci., XLII-2/W9, 639-646, 2019 https://doi.org/10.5194/isprsarchives-XLII-2-W9-639-2019.

Rufian Fdez, F. J., Fernandez Diaz, M., Sabrine, I., Ibanez, J.J., Claramunt-Lopez, B., Escobar, A., Gonzalez Zarandona, J.A., The documentation and protection of cultural heritage during emergencies, Int. Arch. Photogramm. Remote Sens. Spat. Inf. Sci., Volume XLIV-M-1-2020, 2020 HERITAGE2020 (3DPast|RISK-Terra) https://doi.org/10.5194/isprs-archivesXLIV-M-1-2020-287-2020.

Sedláček, J., Klepárník, R., Kopřivová, I., 2020. When does the point cloud become a real tool for a landscape architect? Teaching experience with bachelor and master student programmes in landscape architecture. J. Digit. Landsc. Archit. 2020, 254-261. https://doi.org/10.14627/537690027.

Shults, R., Levin, E., Habibi, R., Shenoy, S., Honcheruk, O., Hart, T., An, Z. 2019. Capability of Matterport 3D camera for industrial archaeology sites inventory, Int. Arch. Photogramm. Remote Sens. Spat. Inf. Sci., Volume XLII-2/W11, 2019 GEORES 2019 - 2nd International Conference of Geomatics and Restoration, 8-10 May 2019, Milan, Italy https://doi.org/10.5194/isprs-archives-XLII-2-W11-1059-2019.

Sulaiman, M., Aziz, M., Bakar, M., Halili, N., Azuddin, M., 2020. Matterport: Virtual Tour as A New Marketing Approach in
Real Estate Business During Pandemic COVID-19, Advances in Social Science, Education and Humanities Research, Vol. 502 Proceedings of the International Conference of Innovation in Media and Visual Design (IMDES 2020).

UNESCO, 2014. Reference Manual. Managing Disaster Risks for World Heritage https://whc.unesco.org/en/managingdisasterrisks/ (June 2021).

Villanueva, J.K.S., Blanco, A.C., 2019. Optimization of ground control point (GCP) configuration for unmanned aerial vehicle (UAV) survey using structure from motion (SFM). Int. Arch. Photogramm. Remote Sens. Spat. Inf. Sci. 42, 167-174. https://doi.org/10.5194/isprs-archives-XLII-4-W12-167-2019. 